Reduktion und Beseitigung des Nitrits ermöglicht, nämlich das Licht.

Die durch höhere Nitritkonzentrationen induzierte Atmungssteigerung hat noch eine weitere Folge: Im geeigneten $p_{\mathrm{H}}$-Bereich (etwa $p_{\mathrm{H}} 3-3,5$ ) bleibt die Atmung (Abb. 2) bei nicht übermäßig starker Nitritanhäufung für mehr als $24 \mathrm{Stdn}$. um über $100 \%$ erhöht. Dann sinkt sie ab, und der RQ erreicht Werte von etwa $-0,7$, eine Erscheinung, die allgemein als Zeichen eines extremen Hungerzustandes, einer Veratmung von Proteinen und plasmatischen Lipoiden, angesehen wird. Offenbar sind dann durch die extrem gesteigerte Atmung die Kohlenhydratreser-

12 Vgl. C. S. French, H. J. Kohn u. P. S. Tang, J. gen. Physiol. 18, 193 [1934].

13 Vgl. M. Cramer u. J. Myers, Plant Physiol. 24, 255 [1949]. ven der Zellen, die normalerweise viel länger ausreichen, erschöpft, so daß bereits frühzeitig ein Hungerstoffwechsel einsetzt ${ }^{12}$. Diese Veränderung des $\mathrm{RQ}$ ist nicht durch $\mathrm{CO}_{2}$-Retention infolge eines starken $p_{\mathrm{H}}$-Anstiegs vorgetäuscht ${ }^{13}$.

Die ausführliche Besprechung dieser Ergebnisse und weiterer Untersuchungen über die Einwirkung äußerer Faktoren (Licht, Vorbelichtung, Kohlenhydrat usw.) auf die beiden ersten Teilprozesse der Nitratreduktion sowie über $p_{\mathrm{H}}$-abhängige Nitritanhäufung bei einigen anderen Grünalgen und ihre Beziehung zur $p_{\mathrm{H}}$-Toleranz wird an anderer Stelle erfolgen.

Die Arbeit wurde im Botanischen Institut der Universität Marburg unter Leitung von Herrn Professor Dr. A. P ir s o n ausgeführt.

\title{
Elektronenmikroskopische Untersuchungen über die Struktur des Hämocyanins von Helix pomatia
}

\author{
Von Gerhard Schramm und Georg Berger \\ Aus dem Max-Planck-Institut für Biochemie, Abt. Virusforschung, Tübingen \\ (Z. Naturforschg. 7 b, 234-287 [1952]; eingegangen am 31. März 1952)
}

Herrn Professor Heinrich Hörlein zum 70. Geburtstag

\begin{abstract}
Die Größe des Hämocyanin-Moleküls und seiner Spaltprodukte wurde elektronenoptisch vermessen. Die erste Dissoziationsstufe besteht in einer Längsspaltung, die entstehenden halben Moleküle sinđ stäbchenförmig mit einer Länge von $40 \mathrm{~m} \mu$ und einem Durchmesser von $12 \mathrm{~m} \mu$. Die weitere Dissoziation erfolgt wahrscheinlich durch Querspaltung dieser Stäbchen in je vier annähernd kugelförmige Untereinheiten.
\end{abstract}

$V_{\text {ans }}$ on Svedberg und Mitarbeitern ${ }^{1}$ wurde gezeigt, daß das Hämocyanin (HC) der Weinbergschnecke (Helix pomatia) in Abhängigkeit vom $p_{\mathrm{H}}$ und von der Elektrolytkonzentration reversibel in Untereinheiten dissoziieren kann, deren Molgewicht die Hälfte bzw. ein Achtel des ursprünglichen beträgt. Die Molekulargewichte wurden von $\mathrm{Brohult}^{2}$ in der Ultrazentrifuge bestimmt. In Tab. 1 sind diese zusammen mit den Reibungsfaktoren $f / f_{0}$ ( $f$ : Reibung des Teilchens, $f_{0}$ : Reibung einer gleichgroßen kompakten Kugel) wiedergegeben. Aus den Reibungsfaktoren wurde unter Vernachlässigung der Hydratation die Länge $L$ berechnet sowie der Durchmesser der Teilchen. In der letzten Spalte ist die Länge der

1 T.Svedberg $\quad$ u. K. O. P ed ersen, Die Ultrazentrifuge (Steinkopff 1940), S. 327.

2 S. Brohult, Nova Acta Reg. Soc. Sci: Upsaliensis [4] $12, \cdot$ Nr. 4 [1940].
Moleküle aufgeführt, wie sie sich aus den Messungen der Strömungsdoppelbrechung von $\mathrm{S}$ n e $11 \mathrm{~m}$ a n

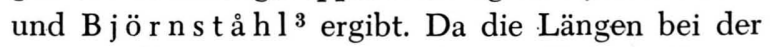
Dissoziation unverändert bleiben, sollte bei diesen Messungen die Dissoziation durch eine Längsspaltung des Moleküls erfolgen. Trurnit und Bergold ${ }^{4}$ versuchten die Form der Dissoziationsprodukte durch Dickenmessung monomolekularer Schichten des HC zu bestimmen. Sie fanden Schichtdicken zwischen 3,8 und $7,1 \mathrm{~m} \mu$ und glaubten ebenfalls, die Dissoziation durch eine Längsspaltung erklären zu können. Als Form schlagen sie für die ganzen Moleküle ein Prisma vor mit einer Grundfläche von $7,5 \times 15 \mathrm{~m} \mu$ und einer Länge von $89 \mathrm{~m} \mu$, für die Achtelmoleküle die gleiche

3 O.Sne $1 \mathrm{~lm}$ a n u. Y. B jörnst å h l, Kolloid-Beihefte 52, 403 [1941].

4 H. J. Trurnit u. G. B ergold, Kolloid-Z. 100, 177 [1942]. 


\begin{tabular}{|l|c|c|c|c|c|c|}
\hline & $M$ & $f / f_{0}$ & \multicolumn{2}{|c|}{ Aus $f / f_{0}$} & \multicolumn{2}{c|}{ Aus St Br } \\
& & & $L$ & $d$ & $L$ \\
\hline & & & & \\
Ganze Moleküle & $\overline{8}, 91 \cdot 10^{6}$ & 1,45 & $113 \mathrm{~m} \mu$ & $13,6 \mathrm{~m} \mu$ & $89 \mathrm{~m} \mu$ & $12,5 \mathrm{~m} \mu$ \\
Halbe Moleküle & 4,31 & 1,40 & 82 & 11,1 & 89 & $8,7 \cdot$ \\
Achtelmoleküle & 1,03 & 1,79 & 82 & 5,4 & 96 & 4,1 \\
\hline
\end{tabular}

$M$ : Molekulargewicht $-f / f_{0}$ : Reibungsverhältnis - L : Länge $-d$ : Durchmesser $-\mathrm{St} \mathrm{Br}$ : Strömungsdoppelbrechung

Tab. 1. Molekulargewicht und Dimensionen von Hämocyanin (Helix pomatia) und seinen Dissoziationsprodukten.

Länge, jedoch eine quadratische Grundfläche mit einer Kantenlänge von $3,75 \mathrm{~m} \mu$. Die theoretischen Grundlagen für die Berechnung der Formkonstanten des HC aus den physikalisch-chemischen Messungen sind unsicher. Eine Nachprüfung im Elektronenmikroskop ist daher wünschenswert. Sie wurde bereits 1946 von B rohult ${ }^{5}$ versucht, doch gelang es ihm bei dem damaligen Stand der Technik nicht, zu reproduzierbaren Ergebnissen zu kommen. Im Zusammenhang mit anderen elektronenmikroskopischen Untersuchungen von Proteinen wurde das Problem von uns wieder aufgegriffen. Unsere Erfahrungen am Tabakmosaikvirus ${ }^{6}$ hatten gezeigt, daß bei der Präparation von Eiweißstoffen besondere Vorsichtsmaßregeln $\mathrm{zu}$ beachten sind, wenn man zu brauchbaren Ergebnissen gelangen will. Beim Eintrocknen in salzhaltigen Lösungen können Proteine sehr leicht in Bruchstücke zerfallen. Die Schwierigkeiten bei der Untersuchung des HC liegen vor allem in der großen Empfindlichkeit gegenüber Salz- und $p_{\mathrm{H}}$-Einflüssen und weiterhin in der geringen Größe der Teilchen, die der Auflösungsgrenze des Elektronenmikroskops nahekommt.

Wir benutzen für unsere Untersuchungen das elektrostatische Elektronenmikroskop von AEG-Zeiß. Nach Kontrollversuchen liegt das Auflösungsvermögen unter den gewählten Bedingungen bei $3 \mathrm{~m} \mu$. Besondere Sorgfalt verlangt die Bedampfung, die mit Pd durchgeführt wurde. Nur wenn die Metallschicht eine sehr kleine Korngröße aufweist, läßt sich die Innenstruktur der HC-Moleküle erkennen. Es zeigte sich ferner, daß die Feinheiten beim Eintrocknen besser erhalten bleiben, wenn man die Teilchen vor der Metallbedampfung mit $\mathrm{OsO}_{4}$-Dampf behandelt. Die besten Aufnahmen aus einer größeren Serie sind in $\mathrm{Abb} .1$ und $2^{*}$ wiedergegeben.

Die unzerfallenen Moleküle besitzen etwa die Gestalt eines Quaders, der durch eine Längsfurche in zwei stäbchenförmige Hälften gespalten ist. Das Molekül hat also die Form der in Süddeutschland üblichen Semmeln.

5 S. Brohult, J. physic. Colloid Chem. 51, 206 [1947].

* Abb. 1, 2 und 4, s. Tafel, S. 288 a.
Die Längsfurche ist bei den einzelnen Molekülen mehr oder weniger ausgeprägt, im Grenzfall liegen die Stäbchen getrennt nebeneinander. Die erste Dissoziationsstufe darf daher entsprechend den älteren Ergebnissen als Längsspaltung aufgefaßt werden. Unsere elektronenmikroskopischen Aufnahmen bestätigen den von $\mathrm{Brohult} \mathrm{t}^{5}$ aus Untersuchungen mit der Ultrazentrifuge erschlossenen Mechanismus der Dissoziation. Der Abstand zwischen den beiden Hälften wird durch Quellung vergrößert, die Bin-

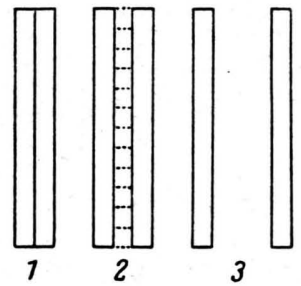

Abb. 3. Quellung und Längsspaltung nach B rohult.

dungskräfte genügen zunächst noch, um das Molekül zusammenzuhalten, bis es schließlich auseinanderfällt (s. Abb. 3).

Die Art der Dissoziation der Halbmoleküle kann elektronenoptisch nicht eindeutig festgestellt werden. Es scheint uns jedoch auf jeden Fall ausgeschlossen, daß diese in einer reinen Längsspaltung besteht. Hiergegen spricht zunächst die Form der isolierten Achtelmoleküle. Durch $p_{\mathrm{H}}$-Verschiebung in das saure oder alkalische Gebiet gelang es, diese Achtelmoleküle in isolierter Form abzubilden. Abb. $4^{*}$ zeigt, daß sie annähernd isodiametrisch und nicht stäbchenförmig sind, wie es bei einer Längsspaltung der Halbmoleküle zu erwarten wäre. Ferner zeigen die undissoziierten Halbmoleküle quer zur Achse Einschnürungen, die auf einen Aufbau aus kugelförmigen Untereinheiten hinweisen. Das Auflösungsvermögen des Elektronenmikroskops reicht nicht aus, die Zahl und die Anordnung dieser Untereinheiten genau festzulegen. Man sieht häufig drei, in günstigen Fällen aber

6 G. S chram m u. M. Wi ed e m a n n, Z. Naturforschg. 6 b, 379 [1951]. 
auch vier linear aneinander gelagerte Partikel. Am wahrscheinlichsten erscheint uns daher die in Abb. $5 \mathrm{a}$ schematisch wiedergegebene Struktur des HC-Moleküls. Daneben ist aber auch eine Anordnung nach Abb. 5 b in Betracht zu ziehen. In diesem Fall würde die Dissoziation der Halbmoleküle in einer Querund Längsspaltung bestehen.

Die elektronenmikroskopischen Abmessungen des Moleküls sind in Abb. 5 b angegeben. Die Länge beträgt demnach $40 \mathrm{~m} \mu$. Dieser Wert wurde auch auf unbedampften Aufnahmen gefunden, bei denen allerdings keine Innenstruktur zu erkennen ist. Den Meßfehler schätzen wir auf 5\%. Die Dicke der Zylinder läßt sich weniger genau angeben, hier dürfte der a

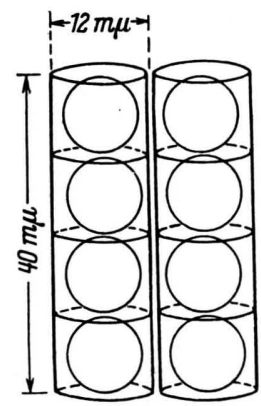

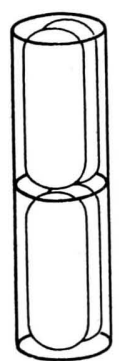

$\mathrm{b}$
Abb. 5. Schema des Aufbaus des Hämocyanins; a) Gesamtmolekül, b) Halbmolekül.

Meßfehler $10 \%$ betragen. Der Mittelwert liegt bei $12 \mathrm{~m} \mu$. Aus den räumlichen Abmessungen des Modells und dem spezifischen Volumen von 0,74 ergibt sich das Gesamtgewicht des $\mathrm{HC}$ zu $7 \cdot 10^{6}$, was mit den Ergebnissen in der Ultrazentrifuge befriedigend übereinstimmt. Auch die Form des Gesamtmoleküls im Elektronenmikroskop ist mit dem in der Ultrazentrifuge gemessenen Reibungsfaktor zu vereinbaren, wenn man die Unsicherheit hinsichtlich der $\mathrm{Hy}$ dratation berücksichtigt.

Die annähernd kugelförmige Gestalt der Achtelmoleküle scheint zunächst mit dem verhältnismäßig hohen Reibungsfaktor und der starken Strömungsdoppelbrechung nicht vereinbar. Aus der Strömungsdoppelbrechung berechnet sich für alle Komponenten eine $\mathrm{zu}$ hohe Asymmetrie. Dies ist vielleicht auf Längsassoziation der gelösten HC-Moleküle zurückzuführen, wofür sich Anhaltspunkte sowohl aus den elektronenmikroskopischen Untersuchungen als auch aus der von $\mathrm{Kratky}^{7}$ am HC durchgeführten Mes-

7 O. Kratky, A. Sekora u. H. FriedrichFreksa, Anzeiger d. Akademie der Wissenschaften in Wien, naturw.-math. Klasse, 1946, Nr. 5, S. 30. sungen der Kleinwinkelstreuung ergeben. Eine solche Assoziation kann jedoch für den hohen Reibungsfaktor in der Ultrazentrifuge kaum verantwortlich gemacht werden. Dieser könnte auch auf einer Ungleichmäßigkeit der Oberfläche oder einer besonders starken Hydratation beruhen. Wir führten Dissoziationsversuche bei verschiedenem $\dot{p}_{\mathrm{H}}$ und verschiedener Salzkonzentration durch. Es werden neben dem ganzen und dem Achtelmolekül auch Zwischenstufen beobachtet, bei denen die regelmäßige Anordnung gestört und ein Teil der Untereinheiten abgebröckelt ist. Bei Gegenwart von Salz fanden wir ähnliche unregelmäßige Gebilde, wie sie von $\mathrm{Brohult} \mathrm{t}^{5}$ in seiner Arbeit in Abb. 1 wiedergegeben sind. Lange Fäden, wie er sie in einem Fall erhielt, konnten auch von uns nicht festgestellt werden.

Von $\mathrm{P}$ ols o n und $\mathrm{W}$ y c k of $\mathrm{f}^{8}$ wurde elektronenoptisch beim Hämocyanin von Busycon canaliculatum ebenfalls eine Innenstruktur beobachtet. Sie unterscheidet sich von der des Hämocyanins aus Helix pomatia, was zu erwarten ist, da das Busycon-Hämocyanin nach Untersuchungen in der Ultrazentrifuge ein anderes Molgewicht und andere Dissoziationsstufen aufweist. Die Gliederung des Hämocyanins wird nicht durch die Fixierung vorgetäuscht, da auch unfixierte Moleküle sie aufweisen. Doch sind deren Umrisse im ganzen mehr verwaschen (s. Abb. $6^{*}$ ).

\section{M e thodik}

Präparation: Zur Gewinnnung des HC wurde den Schnecken das Blut in der üblichen Weise entnommen, dieses sorgfältig dialysiert und die vorhandenen Trübungen abzentrifugiert. Für die Aufnahmen wurden die Lösungen auf eine Proteinkonzentration von etwa $10^{-5} \mathrm{~g} / \mathrm{cm}^{3}$ verdünnt. Es ist wichtig, nur möglichst frische Präparate $\mathrm{zu}$ verwenden, bei älteren ist die Innenstruktur häufig nicht zu erkennen. Wahrscheinlich ist dies darauf zurückzuführen, daß niedrigmolekulare Zerfallsprodukte die Längsfurche verschmieren. Reinigt man solche Lösungen durch Ausschleudern auf der Ultrazentrifuge, so wird die Innenstruktur wieder sichtbar, sie ist aber meist nicht so deutlich wie bei frischen Präparaten. Bei Untersuchungen in Pufferlösungen wurde das Salz vor dem Eintrocknen des Tröpfchens wiederholt mit destilliertem Wasser ausgewaschen. Gute Aufnahmen der Achtelmoleküle wurden in $m / 10$-Phosphat vom $p_{\mathrm{H}} 7,8$ erhalten.

Fix i e r u n g : Zur Fixierung werden die auf die Folie gebrachten Tröpfchen der HC-Lösung $3 \mathrm{Min}$. in einem geschlossenen Behälter den Dämpfen einer 2,5-proz. Osmiumsäure ausgesetzt. Je nach Art der Fixierungsbedin-

8 A. P ols on u. R. W. G. W y ck of f, Nature [London] 160, 153 [1947].

* Abb. 6, s. Tafel, S. 288 a. 
gungen erhält man ganze oder Achtelmoleküle. Wählt man große Tröpfchen, so löst sich verhältnismäßig viel $\mathrm{OsO}_{4}$ in ihnen, dies hat beim nachfolgenden Eintrocknen eine $p_{\mathrm{H}}$-Erniedrigung zur Folge, die zu einer Dissoziation führt (s. Abb. 4). Läßt man die aufgebrachten Tröpfchen erst $3 \mathrm{Min}$. eintrocknen und bringt sie dann in $\mathrm{OsO}_{4}$ Dampf, so erhält man die ganzen Moleküle, da in diesem Fall die $p_{\mathrm{H}^{-}}$-Verschiebung gering ist (Abb. $1 \mathrm{u}$. 2).

B e d a m p f ung: Es wurde mit einer möglichst geringen Menge von Pd unter einem Winkel von 25 bis $30^{\circ}$ bedampft, wobei das Metall langsam erhitzt wurde. Ein guter gleichmäßiger Untergrund war nur auf frisch bedampften Folien zu beobachten. Bereits nach wenigen Tagen wurde die Metallschicht grobkörnig. Präparate, die schon einmal den Elektronenstrahlen ausgesetzt waren, erwiesen sich bereits am Tage nach der ersten Aufnahme als unbrauchbar. Bei höherer Intensität der Bestrahlung beobachteten wir auch schon während der Aufnahme Veränderungen des Untergrunds.

A uf nahmetechnik: Um diese Veränderungen zu verhindern und um möglichst hohe Kontraste zu erhalten, wurde bei schwacher Intensität und geringer Strahlspannung gearbeitet $(35 \mathrm{kV})$. Hierdurch wird allerdings die Scharfstellung und die genaue Korrektur des Astigmatismus sehr erschwert, die bei der Kleinheit der untersuchten Objekte sehr sorgfältig sein müssen. Die elektronenoptische Vergrößerung betrug $1: 15000$, anschließend wurde auf 1:90000 nachvergrößert. Die Eichung des Vergrößerungsfaktors wurde bereits an anderer Stelle ${ }^{6}$ beschrieben.

\title{
Bestimmungen des Tryptophangehaltes verschiedener Rassen der Mehlmotte Ephestia Kühniella als Beitrag zur Analyse der Genwirkungen
}

\author{
Von Adolf Butenandt und Wolfgang Albrecht \\ Aus dem Max-Planck-Institut für Biochemie \\ und dem Physiol.-chem. Institut der Universität, Tübingen \\ (Z. Naturforschg. 7 b, 287-290 [1952]; eingegangen am 24. April 1952) \\ Heinrich Hörlein zum 70. Geburtstag
}

\begin{abstract}
In Bestätigung der Befunde von E. C a s p a r i wird nachgewiesen, daß die Proteine der Larven und Motten von aa-Ephestia-Mutanten einen signifikant höheren Gehalt an Tryptophan enthalten als die der $a+a+$-Wildform. Dieser erhöhte Tryptophangehalt ist bei $a a$-Mutanten mit verschiedener Augenfarbnuance von gleicher Größenordnung und wird durch Unterschiede in der Tryptophankonzentration des Futters nicht wesentlich beeinflußt. Die sich aus diesen Befunden ergebenden Konsequenzen für die zur Diskussion stehenden Vorstellungen über das Wesen der Genwirkungen werden besprochen.
\end{abstract}

$\mathrm{D}$ ie Augenpigmente (Ommochrome) der Mehlmotte Ephestia und der Taufliege Drosophila werden in einer durch mehrere Gene gesteuerten Reaktionskette aus Tryptophan gebildet ${ }^{1}$; als Zwischenprodukte treten dabei die Chromogene Kynurenin ${ }^{2,3}$ und 3 -Oxy-kynurenin ${ }^{4}$ auf.

Bei der Mutante $a$ der Ephestia und der Mutante $v$ der Drosophila unterbleibt die Ommochrombildung, weil die zum Pigment führende Reaktionskette unterbrochen ist; die Mutanten haben die Fähigkeit der Wildform eingebüßt, Tryptophan oxydativ in Kynurenin überzuführen. Dieser 1940 erhobene Befund führte uns zu der Hypothese, daß ein Gen seine

1 A. B ute n a ndt, Angew. Chem. 61, 262 [1949]; Jb. Max-Planck-Gesellschaft 1951, 160.

2 A. B u.te nand $t$, W. We id e l u. E. B e c ker, Naturwiss. 28, 63, 447 [1940]. Zur Vorgeschichte der GenFerment-Hypothese vgl. G. W. B e a d le in „L.C. D u n n, Genetics in the 20th Century “. Macmillan Co., New York 1951, S. 223.
Wirkung über die Bereitstellung eines spezifischen Fermentes entfalte ${ }^{2}$; die durch eine Genmutation bedingte Unterbrechung einer biochemischen Reaktionskette könnte auf Grund dieser Vorstellung dadurch verursacht sein, daß ein dem Normalgen zuzuordnendes spezifisches Ferment nicht mehr ausreichend gebildet oder daß es in seiner Struktur verändert wird. Die Hypothese von der Wirkung der Gene über die Bereitstellung spezifischer Fermente hat sich in der Folge als äußerst fruchtbar erwiesen, insbesondere bei der Aufklärung biochemischer Reaktionsketten mit Hilfe von Mutanten des Schimmelpilzes Neurospora crassa ${ }^{5}$.

${ }^{3}$ A. But enand t, W. Weidel, R. Weichert u. W. v. Derjugin, Hoppe-Seyler's Z. physiol. Chem. 279, 27 [1943].

4 A. B u te nandt, W. Weidel u. H. S chloßb e r g e r, Z. Naturforschg. 4 b, 242 [1949].

5 G. W. B e a d l e, Annu. Rev. Physiol. 10, 17 [1948]; Fortschr. Chem. organ. Naturstoffe 5, 300 [1948]. 